\title{
Avaliação de parâmetros fisico-hídricos de Latossolo Vermelho sob pastejo e sob cerrado
}

\author{
Thiago A. Borges ${ }^{1}$, Fernanda A. Oliveira ${ }^{1}$, Euzebio M. da Silva ${ }^{1} \&$ Wenceslau J. Goedert ${ }^{2}$
}

\begin{abstract}
RESUMO
O objetivo primeiro neste trabalho foi avaliar a qualidade fisico-hídrica do solo em áreas sob pastejo, em relação a um cerrado natural. Os tratamentos foram: cerrado natural, cerrado antropizado (pastejado na estação seca) e três pastagens cultivadas, com diferentes graus de cobertura do solo. O estudo foi realizado na Embrapa Cerrados em um Latossolo Vermelho, nas camadas de 0-5, 10-15, 40-55 e 70-80 cm, cujos parâmetros avaliados foram condutividade hidráulica saturada (Ks), capacidade de água disponível (CAD), densidade do solo (Ds) e macroporosidade (Ma). A qualidade fisicohídrica do solo nas áreas sob pastejo foi analisada tendo-se como referência os parâmetros medidos no cerrado natural. Observou-se semelhança estatística da CAD na camada 0-5 cm para todas as áreas mas ocorreu drástica redução da saturação efetiva nas áreas sob pastejo, explicada principalmente pela queda na macroporosidade. A redução média da Ma foi de 70,8\%, ocasionando um decréscimo da ordem de 73,6\% da Ks nos primeiros $15 \mathrm{~cm}$ das áreas sob pastejo, podendo-se concluir que a camada superficial do solo foi a mais afetada pelo pastejo, com perda significativa da qualidade fisico-hídrica e previsível redução na capacidade de infiltração da água no solo.
\end{abstract}

Palavras-chave: sustentabilidade, condutividade hidráulica saturada, capacidade de água disponível, Brachiaria decumbens

\section{Evaluation of soil-water parameters of a Red Latosol under pasture and 'cerrado'}

\begin{abstract}
The objective of this study was to evaluate the soil-water quality under grazing pastures in comparison to a natural 'cerrado' (savanna). The treatments were: natural 'cerrado', grazed 'cerrado' during the dry season, and three cultivated pastures with different degrees of soil covering. The research was conducted at the Embrapa Cerrados, on a Red Latosol in the 0-5, 10-15, 40-55, and 70-80 cm soil layers, in which the following parameters were quantified: saturated hydraulic conductivity (Ks), available water capacity (CAD), soil bulk density (Ds), and macroporosity (Ma). The soil-water quality was determined by taking as references the corresponding parameters measured in the natural 'cerrado'. Statistical similarity of CAD values in the $0-5 \mathrm{~cm}$ soil layer for all treatments was observed. However, there was a drastic reduction of the effective saturation in the treatments of grazed areas, mainly explained by the reduction in macroporosity. The mean decrease in Ma was $70.8 \%$, causing a $73.6 \%$ decline in $\mathrm{Ks}$ in the $15 \mathrm{~cm}$ upper soil layer of the grazed areas, which led to the conclusion that the surface soil layers were the most affected by the grazing activity, with significant deterioration of their soil-water quality and a predictable reduction in water-infiltration capacity.
\end{abstract}

Key words: sustainability, saturated hydraulic conductivity, available water capacity, Brachiaria decumbens

Embrapa Cerrados, BR 020, km 18, CP 08223,CEP 73310-970, Planaltina, DF. Fone: (61) 3388-9803.E-mail: thiago.alvesborges@gmail.com; abreu.fernanda@uol.com.br; euzebio@cpac.embrapa.br

2 Faculdade de Agronomia e Medicina Veterinária/UnB, CP 04508, CEP 70919-970, Brasília, DF. Fone: (61) 3307-2823, ramal 2322. E-mail: goedert@unb.br 


\section{INTRODUÇÃO}

O cerrado é considerado o segundo maior bioma da América Latina, com cerca de 204 milhões de hectares com mais de $50 \%$ de sua área coberta com pastagens cultivadas e naturais no ano 2000 (Sano et al., 2000); entretanto, a situação na qual se encontram essas pastagens é preocupante, já que cerca de $80 \%$ delas apresentam algum grau de degradação (Barcellos, 1996).

Dependendo da intensidade do manejo adotado em curto período de tempo ( 3 a 5 anos) as pastagens podem apresentar cobertura vegetal deficiente, deixando o solo exposto diretamente aos raios solares, às intempéries, ao escoamento superficial da água e ao pisoteio dos animais (Balbino et al., 2003). Desta maneira se abre caminho para a deterioração da estrutura do solo, redução da sua capacidade produtiva, formação de camadas compactadas, aumento da susceptibilidade à erosão e maior incidência de problemas fitossanitários.

A deterioração das propriedades físicas é um processo que se inicia tão logo um manejo inadequado é adotado; contudo, somente lhe é dada devida importância nas etapas finais do processo de degradação das pastagens, requerendo medidas mais drásticas e onerosas para a recuperação do sistema produtivo.

A atividade agropecuária tende a modificar a porosidade do solo, devido principalmente aos processos de compactação e adensamento, que conduzem ao incremento dos valores de densidade do solo. Silva \& Kato (1997) verificaram a superioridade dos valores de macroporosidade e de condutividade hidráulica em um cerrado virgem em relação aos sistemas de plantio direto e de plantio convencional, ratificando a influência decisiva da macroporosidade sob os valores de condutividade hidráulica. Nesses aspectos, a avaliação de propriedades físicas de solo se torna importante ferramenta no monitoramento da qualidade ambiental, considerando-se as características e finalidade do uso de determinado agroecossistema (Dias, 2002).

Propôs-se, neste trabalho, avaliar a qualidade fisico-hídrica de um Latossolo Vermelho sob pastejo rotacionado de Brachiaria decumbens cv. Basilisk, em relação a um cerrado natural, no Distrito Federal.

\section{MATERIAL E MÉTODOS}

As amostras de solo foram coletadas em fevereiro de 2005, no campo experimental 'Chapadão' da Embrapa Cerrados, Planaltina, DF, com altitude de $1.200 \mathrm{~m}$, relevo plano a suave-ondulado, fase Cerrado Típico e limitada pelas coordenadas geográficas: $15^{\circ} 38^{\prime} 45^{\prime \prime}$ a $15^{\circ} 38^{\prime}$ '53” de latitude sul e $47^{\circ} 45^{\prime} 15^{\prime}$ ' a $47^{\circ} 44^{\prime} 41^{\prime}$ ' de longitude oeste. O solo está classificado como Latossolo Vermelho Distrófico A moderado, segundo o Sistema Brasileiro de Classificação de Solos (EMBRAPA, 1999), com textura argilosa e valores médios aproximados de $580 \mathrm{~g} \mathrm{~kg}^{-1}$ de argila, $90 \mathrm{~g} \mathrm{~kg}^{-1}$ de silte e $330 \mathrm{~g} \mathrm{~kg}^{-1}$ de areia total. De acordo com Köppen, o clima da região é classificado como tropical estacional de savana
(Aw), com temperatura média anual em torno de $22^{\circ} \mathrm{C}$ e duas estações bem definidas: a seca e a chuvosa.

Selecionaram-se na área experimental cinco tipos de cobertura vegetal: uma área com vegetação de cerrado natural (Cn); uma de cerrado antropizado (Ca) e três áreas com pastagens cultivadas de Brachiaria decumbens cv. Basilisk, com diferentes graus de cobertura do solo, denominadas: pastagem com alta cobertura (Pac), pastagem com média cobertura (Pmc) e pastagem com baixa cobertura (Pbc). A área ‘cerrado antropizado' apresentava faixas contínuas de vegetação de cerrado strictu sensu intercaladas com faixas desmatadas nas quais se formou uma área vegetada com gramíneas espontâneas, que serviam de alimento suplementar ao gado durante a estação seca. A vegetação do 'cerrado natural' também foi caracterizada como cerrado strictu sensu, sendo que seu histórico não apresentava relato de atividade antrópica local.

A área de cada pastagem cultivada era de 0,75 ha e o grau de cobertura do solo, nessas áreas, definido de acordo com a produtividade das pastagens, as quais vinham sendo cultivadas desde 2002, a níveis diferenciados de adubação. A Pbc não recebeu adubação de manutenção, sendo que a adubação da Pac e da Pmc se diferenciou pela fonte de nitrogênio e enxofre (Pac usou sulfato de amônio e superfosfato triplo, enquanto Pmc usou uréia e superfosfato simples) e pela dose de fósforo empregada (Pac usou $40 \mathrm{~kg} \mathrm{ha}^{-1}$, enquanto Pmc usou $20 \mathrm{~kg} \mathrm{ha}^{-1}$ ). A massa de forragem média foi de 3,10 kg ha-1 para Pac; de 3,03 kg ha-1 para Pmc e de $1,94 \mathrm{~kg} \mathrm{ha}^{-1}$ para Pbc. O pastejo adotado foi o rotacionado, com períodos de ocupação e descanso de 14 dias e oferta de forragem de 10-11 kg matéria seca por $100 \mathrm{~kg}$ de peso vivo.

Foram analisados os seguintes parâmetros físico-hídricos do solo: densidade do solo (Ds), macroporosidade (Ma), capacidade de água disponível (CAD), curvas de conteúdo de água relativo (cCAR) e condutividade hidráulica saturada de campo (Ks). Para as determinações de laboratório foram coletadas amostras indeformadas do solo, retiradas perpendicularmente ao plano do terreno, em quatro repetições, nas camadas de solo: 0-5, 10-15, 40-55 e 70-80 cm, utilizando-se cilindros metálicos de $5,1 \mathrm{~cm}$ de altura e 5,0 cm de diâmetro. Após a saturação das amostras os cilindros foram pesados e submetidos a centrifugação para determinação de nove pontos de conteúdo gravimétrico de água do solo (Silva et al., 2006), através do método da centrifugação (Silva \& Azevedo, 2002); ao final deste processo, a Ds foi mensurada pelo método do anel volumétrico (EMBRAPA, 1997). O parâmetro Ma foi calculado como sendo a diferença entre os conteúdos gravimétricos de água medidos na saturação e em equilíbrio com a tensão de $6 \mathrm{kPa}$ (EMBRAPA, 1997). A CAD foi definida como a diferença entre os conteúdos gravimétricos de água medidos na tensão de $10 \mathrm{kPa}$, considerada na capacidade de campo (Souza \& Reichardt, 1996), e em equilíbrio com a tensão de $1.520 \mathrm{kPa}$, em torno do qual é normalmente estabelecido o ponto de murcha permanente ou limite inferior do conteúdo de água no solo (Beutler et al., 2004).

Com os conteúdos gravimétricos de água do solo obtidos 
pelo método da centrifugação, calcularam-se os valores do conteúdo de água relativo $\left(\mathrm{CAR}_{\mathrm{i}}\right)$, em cada ponto de tensão aplicado (kPa), utilizando-se a Eq. 1:

$$
\mathrm{CAR}_{\mathrm{i}}=\frac{\theta_{\mathrm{i}}-\theta_{1.520 \mathrm{kPa}}}{\theta_{\text {sat }}-\theta_{1.520 \mathrm{kPa}}}
$$

em que:

$\mathrm{CAR}_{\mathrm{i}}$ - conteúdo de água relativo, variando de 0 a 1 , correspondente ao conteúdo gravimétrico de água no solo $\theta_{\mathrm{i}}$ referente ao ponto de tensão i

$\theta_{\text {sat }}$ - conteúdo gravimétrico de água na saturação

$\theta_{1.520 \mathrm{kPa}}$ - conteúdo gravimétrico residual da água do solo, correspondente à tensão de $1.520 \mathrm{kPa}$

$\theta_{\text {sat }}-\theta_{1.520 \mathrm{kPa}}-$ valor equivalente à saturação efetiva, $\mathrm{S}_{\mathrm{e}}$

Para cada conjunto de valores de $\mathrm{CAR}_{\mathrm{i}}$ se construiu uma curva contínua de conteúdo de água relativo (cCAR), utilizando-se o modelo não-linear (Assouline et al., 1998), expresso por:

$$
\mathrm{cCAR}=1-\left[\exp \left(-\alpha\left(\frac{1}{\mathrm{~T}}-\frac{1}{\mathrm{~T}_{\max }}\right)^{\mathrm{n}}\right)\right]
$$

em que:

cCAR - curva ajustada ao conteúdo de água relativo (CAR)

n e $\alpha$ - parâmetros empíricos obtidos por procedimentos de otimização não linear do programa Microsoft Excel

$\mathrm{T}$ - tensão ( $\mathrm{kPa})$ em um ponto qualquer correspondente ao conteúdo de água relativo do solo

$\mathrm{T}_{\max }$ - tensão equivalente a $1.520 \mathrm{kPa}$, em equilíbrio $\operatorname{com} \theta_{1.520 \mathrm{kPa}}$

A condutividade hidráulica saturada (Ks) de campo foi calculada utilizando-se o permeâmetro de Guelph (Reynolds \& Elrick, 1987), com furos no solo com um raio (R) de $3 \mathrm{~cm}$ e carga hidráulica $(\mathrm{H})$ única de 3 ou $5 \mathrm{~cm}$. As medições foram feitas em seis repetições por camada (10-15, 40-55 e 70-80 cm) em um local representativo de cada tratamento; a camada de 0 a $5 \mathrm{~cm}$ não foi avaliada, pois esse tipo de equipamento requer uma profundidade mínima de furo de aproximadamente 6-10 cm para operar com as cargas hidráulicas adotadas.

Fez-se o cálculo dos valores de Ks (Reynolds \& Elrick, 1987) aplicando-se a Eq. 3 acondicionada da seguinte forma:

$$
\mathrm{Ks}=\frac{\mathrm{CQ}}{2 \pi \mathrm{H}^{2}+\mathrm{C} \pi \mathrm{R}^{2}+2 \pi \frac{\mathrm{H}}{\mathrm{A}}}
$$

em que:

$$
\begin{aligned}
& \text { Ks - a condutividade hidráulica saturada de campo, } \\
& \text { mm h-1 } \\
& \text { Q - vazão média infiltrada, } \mathrm{m}^{3} \mathrm{~s}^{-1} \\
& \mathrm{H} \text { - carga hidráulica, } \mathrm{m} \\
& \mathrm{R} \text { - raio do furo, } \mathrm{m} \\
& \mathrm{A} \text { - parâmetro associado à textura e estrutura do } \\
& \quad \text { solo, } \mathrm{m}^{-1} \\
& \mathrm{C} \text { - fator adimensional, estabelecido em função da } \\
& \text { relação } \mathrm{H} / \mathrm{R}
\end{aligned}
$$

Seguindo-se as recomendações de Reynolds \& Elrick (1987) e se considerando as características de elevada infiltração do Latossolo Vermelho, elegeu-se o valor de $\mathrm{A}=12 \mathrm{~m}^{-1}$; além disso, digitalizando sua relação gráfica entre $\mathrm{C}$ e H/R e se procedendo ao ajuste dos pontos obtidos por meio de regressão não-linear, chegou-se à seguinte Eq. 4 para o cálculo analítico de C:

$$
\mathrm{C}=\mathrm{c}_{1}+\mathrm{c}_{2}\left(\frac{\mathrm{H}}{\mathrm{R}}\right)^{\mathrm{b}}, \quad \text { para } \quad 0,1019 \leq \frac{\mathrm{H}}{\mathrm{R}} \leq 8,9358
$$

em que: $c_{1}, c_{2}$ e b são parâmetros empíricos de ajuste, com valores iguais a $-0,1755,0,7823$ e 0,5108 , respectivamente.

De acordo com Warrick (1983), os valores de Ks seguem a distribuição log-normal, de modo que antes de serem utilizados em procedimentos estatísticos baseados na distribuição normal, eles devem ser transformados logaritmicamente; assim se procedendo, os valores resultantes de logKs foram então submetidos às análises univariadas, aplicando-se o teste $\mathrm{F}$ para comparação de variâncias, seguido do teste t Student para comparação de médias, ambos a 1\% de significância. A estatística utilizada para se comparar as curvas de conteúdo de água relativo (cCAR) foi o método das curvas completas (Silva \& Azevedo, 2002), adotando-se o teste F para a comparação entre as curvas, também a 1\% de significância.

A qualidade físico-hídrica do solo foi avaliada por meio de modelos gráficos, do tipo radial, em que cada parâmetro foi locado em um dos seus raios (Costa et al., 2006). Os parâmetros físico-hídricos medidos no cerrado natural (Cn) foram adotados como referência, já que as condições de uso desse solo possivelmente ainda refletiam o equilíbrio original das áreas estudadas. $\mathrm{O}$ indicador numérico para expressar o efeito do tratamento sobre cada parâmetro avaliado (Ds, Ma, CAD e $\operatorname{logKs}$ ) foi definido como sendo a diferença entre a unidade e o quociente do valor do parâmetro considerado e seu respectivo valor de referência (ambiente cerrado natural), multiplicada por 100; assim, o valor deste indicador numérico de qualidade era sempre zero para o tratamento de referência; positivo para qualquer valor superior ao de referência e negativo para qualquer valor inferior. Com esse tipo de apresentação foi possível construir um esquema gráfico radial para avaliar qualitativamente a tendência do impacto em relação ao uso contínuo dessas áreas na qualidade físico-hídrica do solo.

\section{RESULTADOS E DISCUSSÃO}

Uma análise geral dos dados obtidos (Tabela 1) revela que as principais modificações nos atributos físico-hídricos do solo ocorreram nas camadas superficiais (0-5 e 10-15 cm), para todas as áreas sob uso antrópico.

$\mathrm{O}$ atributo CAD foi o único que se manteve relativamente homogêneo entre as áreas estudadas em qualquer profundidade. As áreas que sofreram as maiores alterações foram aquelas sob pastagem cultivada. Verificou-se, nas camadas mais profundas (40-55 e 70-80 cm) que as variáveis Ds, Ma, CAD, Se e Ks apresentaram, praticamente, o mesmo comportamento nas áreas estudadas, o que permitiu inferir que o pastejo 
Tabela 1. Valores médios ${ }^{1}$ da densidade do solo (Ds), macroporosidade (Ma), capacidade de água disponível (CAD), saturação efetiva (Se) e condutividade hidráulica saturada (Ks)

\begin{tabular}{|c|c|c|c|c|c|c|}
\hline $\begin{array}{l}\text { Parâmetros } \\
\text { (unidade) }\end{array}$ & $\begin{array}{l}\text { Camadas } \\
\text { (cm) }\end{array}$ & $\begin{array}{l}\text { Cerrado natural } \\
\text { (Cn) }\end{array}$ & $\begin{array}{l}\text { Cerrado antropizado } \\
\text { (Ca) }\end{array}$ & $\begin{array}{c}\text { Pastagem com alta } \\
\text { cobertura (Pac) }\end{array}$ & $\begin{array}{l}\text { Pastagem com média } \\
\text { cobertura (Pmc) }\end{array}$ & $\begin{array}{c}\text { Pastagem com baixa } \\
\text { cobertura ( } \mathrm{Pbc})\end{array}$ \\
\hline \multirow{4}{*}{ Ds $\left(\mathrm{g} \mathrm{cm}^{-3}\right)$} & $0-5$ & $0,871 \mathrm{aC}$ & $1,203 \mathrm{aA}$ & $1,147 \mathrm{aAB}$ & $1,102 \mathrm{aB}$ & $1,190 \mathrm{aA}$ \\
\hline & $10-15$ & $0,967 \mathrm{aB}$ & $1,113 \mathrm{bA}$ & 1,139 aA & $1,100 \mathrm{Aa}$ & $1,119 \mathrm{aA}$ \\
\hline & $40-55$ & $0,984 \mathrm{aAB}$ & 1,077 bA & 0,991 bB & $0,969 \mathrm{bB}$ & $1,023 \mathrm{bAB}$ \\
\hline & $70-80$ & $0,919 \mathrm{aBC}$ & $0,997 \mathrm{CAB}$ & $1,036 \mathrm{bA}$ & $0,904 \mathrm{cC}$ & $1,012 \mathrm{bAB}$ \\
\hline \multirow{4}{*}{$\operatorname{Ma}\left(g g^{-1}\right)$} & $0-5$ & $0,299 \mathrm{aA}$ & $0,073 \mathrm{cB}$ & $0,050 \mathrm{cB}$ & $0,023 \mathrm{cD}$ & $0,040 \mathrm{cC}$ \\
\hline & $10-15$ & 0,239 aA & $0,145 \mathrm{bB}$ & 0,089 bC & $0,077 \mathrm{bc}$ & 0,099 bBC \\
\hline & $40-55$ & 0,224 aA & $0,157 \mathrm{bA}$ & $0,173 \mathrm{aA}$ & $0,181 \mathrm{aA}$ & 0,165 aA \\
\hline & $70-80$ & 0,248 aA & 0,222 aA & $0,151 \mathrm{aB}$ & 0,216 aA & $0,182 \mathrm{aAB}$ \\
\hline \multirow{4}{*}{$\operatorname{CAD}\left(g g^{-1}\right)$} & $0-5$ & 0,139 acA & $0,131 \mathrm{aA}$ & $0,148 \mathrm{aA}$ & 0,146 aA & $0,144 \mathrm{aA}$ \\
\hline & $10-15$ & $0,097 \mathrm{cBC}$ & $0,097 \mathrm{aC}$ & 0,116 bAB & 0,114 bcABC & $0,131 \mathrm{aA}$ \\
\hline & $40-55$ & 0,104 сABC & $0,102 \mathrm{aBC}$ & $0,124 \mathrm{bA}$ & $0,097 \mathrm{cC}$ & 0,129 aA \\
\hline & $70-80$ & 0,118 bBC & $0,100 \mathrm{aC}$ & 0,121 bAB & $0,121 \mathrm{bB}$ & $0,129 \mathrm{aA}$ \\
\hline \multirow{4}{*}{ Se $\left(g^{-1}\right)$} & $0-5$ & 0,473 aA & $0,221 \mathrm{cB}$ & $0,208 \mathrm{bB}$ & $0,197 \mathrm{bB}$ & $0,199 \mathrm{cB}$ \\
\hline & $10-15$ & $0,363 \mathrm{bA}$ & $0,262 \mathrm{cB}$ & 0,229 bC & 0,217 bC & $0,252 \mathrm{bBC}$ \\
\hline & $40-55$ & $0,361 \mathrm{abAB}$ & $0,303 \mathrm{bB}$ & 0,351 aA & $0,320 \mathrm{aAB}$ & $0,335 \mathrm{aAB}$ \\
\hline & $70-80$ & $0,402 \mathrm{abA}$ & $0,363 \mathrm{aA}$ & $0,317 \mathrm{aB}$ & $0,373 \mathrm{aA}$ & 0,359 aAB \\
\hline \multirow{4}{*}{$\mathrm{Ks}^{2}\left(\mathrm{~cm} \mathrm{~h}^{-1}\right)$} & $0-5$ & - & - & - & - & - \\
\hline & $10-15$ & $12,124 \mathrm{aA}$ & $6,134 \mathrm{bB}$ & $2,502 \mathrm{cC}$ & $1,899 \mathrm{cC}$ & 2,264 bC \\
\hline & $40-55$ & $11,311 \mathrm{aAB}$ & $19,333 \mathrm{aA}$ & 7,963 bBC & 7,605 bCD & $7,851 \mathrm{aD}$ \\
\hline & $70-80$ & $12,443 \mathrm{aA}$ & $23,137 \mathrm{aA}$ & $18,530 \mathrm{aA}$ & $20,650 \mathrm{aA}$ & 14,896 aA \\
\hline
\end{tabular}

${ }^{1}$ Médias seguidas das letras iguais (maiúsculas nas colunas comparam camadas e minúsculas nas linhas comparam tratamentos) não diferem entre si a $1 \%$ de probabilidade pelo teste t-Student. 20 parâmetro Ks não foi avaliado na camada de $0 \mathrm{a} 5 \mathrm{~cm}$ devido à impossibilidade técnica do tipo usado de Permeâmetro de Guelph não poder ser empregado nessa profundidade nos testes de campo

pouco influenciou a estrutura do solo e, em conseqüência, os padrões de armazenamento e movimentação de água nessas camadas do solo; além dessa dedução, tal resultado levou também à fundamentação prática de que, originalmente, essas áreas apresentavam solos em condições físicas e origens semelhantes, o que torna possível a comparação entre os parâmetros físico-hídricos das camadas superiores e permite que seja atribuída, às atividades de pastejo, a razão das diferenças nas áreas.

O efeito negativo do pastejo em relação à variável Se (Tabela 1) foi mais evidente nas camadas superficiais, produzindo quedas de até 58\% nas áreas com pastagem cultivada em relação à área $\mathrm{Cn}$. A redução da Se e o aumento da CAR a $10 \mathrm{kPa}$, observados nas áreas pastejadas, contrabalancearam e proporcionaram valores de CAD próximos ao patamar de referência deste trabalho (cerrado natural). Conforme a análise estatística deste parâmetro, todas as áreas foram estatisticamente semelhantes em 0-5 cm (média de 0,1416 $\mathrm{g} \mathrm{g}^{-1}$ ) e pouco diferiram em 10-15 cm, com aumento médio de 24,1\% para as pastagens cultivadas.

Em estudos dessa natureza, Fregonezi et al. (2001) concluíram que o efeito da compactação pelo pisoteio animal atinge a microestrutura do solo, com conseqüências negativas sobre a estrutura dos microporos internos dos agregados, reduzindo sua capacidade de armazenamento de água no interior dos grumos. Essas conclusões corroboram com os resultados encontrados neste trabalho, ficando comprovado que, praticamente, não houve aumento do armazenamento de água na camada 0-5 cm do solo das áreas sob pastejo.

Do ponto de vista das diferenças em termos do conteúdo da água do solo, os resultados podem ser avaliados comparando-se as respectivas curvas de retenção as quais, para isto, devem ser construídas sob uma mesma escala de referência (Figura 1), aplicando-se a técnica do escalonamento de propriedades hídricas de solos (Bacchi \& Reichardt, 1988).

Nesta comparação se verifica, graficamente, tendência de aproximação das curvas com o aumento da profundidade, reforçando a idéia da similaridade do solo nas camadas mais profundas. Da análise estatística feita verificou-se, aplicando-se a técnica de comparação de curvas (Silva \& Azevedo, 2002), que as curvas de conteúdo de água relativo do solo sob pastagens cultivadas, das camadas mais superficiais (0-5 e 10-15 cm), não apresentaram diferenças significativas entre si, mas diferiram de Cn e Ca que foram diferentes entre si (Figuras 1A e B). Em geral, nas camadas mais profundas (40-55 e 70-80 cm) não houve diferença significativa entre a média dos tratamentos sob pastagens e da área sob Cn (Figuras 1C e D).

Tomando-se por base o ponto de tensão de $10 \mathrm{kPa}$ (Figura $1 \mathrm{~A}, \mathrm{~B}, \mathrm{C}$ e D), verificou-se que houve aumento relativo no armazenamento de água no solo das áreas sob pastejo em relação a Cn para as camadas superiores. Por exemplo, os valores de CAR a $10 \mathrm{kPa}$, na camada $0-5 \mathrm{~cm}$ (Figura 1A), giraram em torno de 60 e de $70 \%$, respectivamente, para Ca e para as pastagens cultivadas; e de 40 e de $50 \%$ para as mesmas áreas, na camada de 10-15 cm (Figura 1B); os valores de CAR na área Cn foram da ordem de $30 \%$, em ambas as camadas.

A retenção de água nas camadas superficiais do solo das áreas sob pastejo pode ser interpretada a partir dos valores de Se e Ma. Na camada de $0-5 \mathrm{~cm}$, a Se das áreas sob pastejo teve uma queda média de 56,5\%; já na camada de 10-15 cm essa redução foi da ordem de 34\% em média. Tais diferenças foram essencialmente provocadas pela redução da macroporosidade, tendo quase nenhuma alteração significativa na microporosidade (Tabela 1). Esses resultados mostram drásticas 


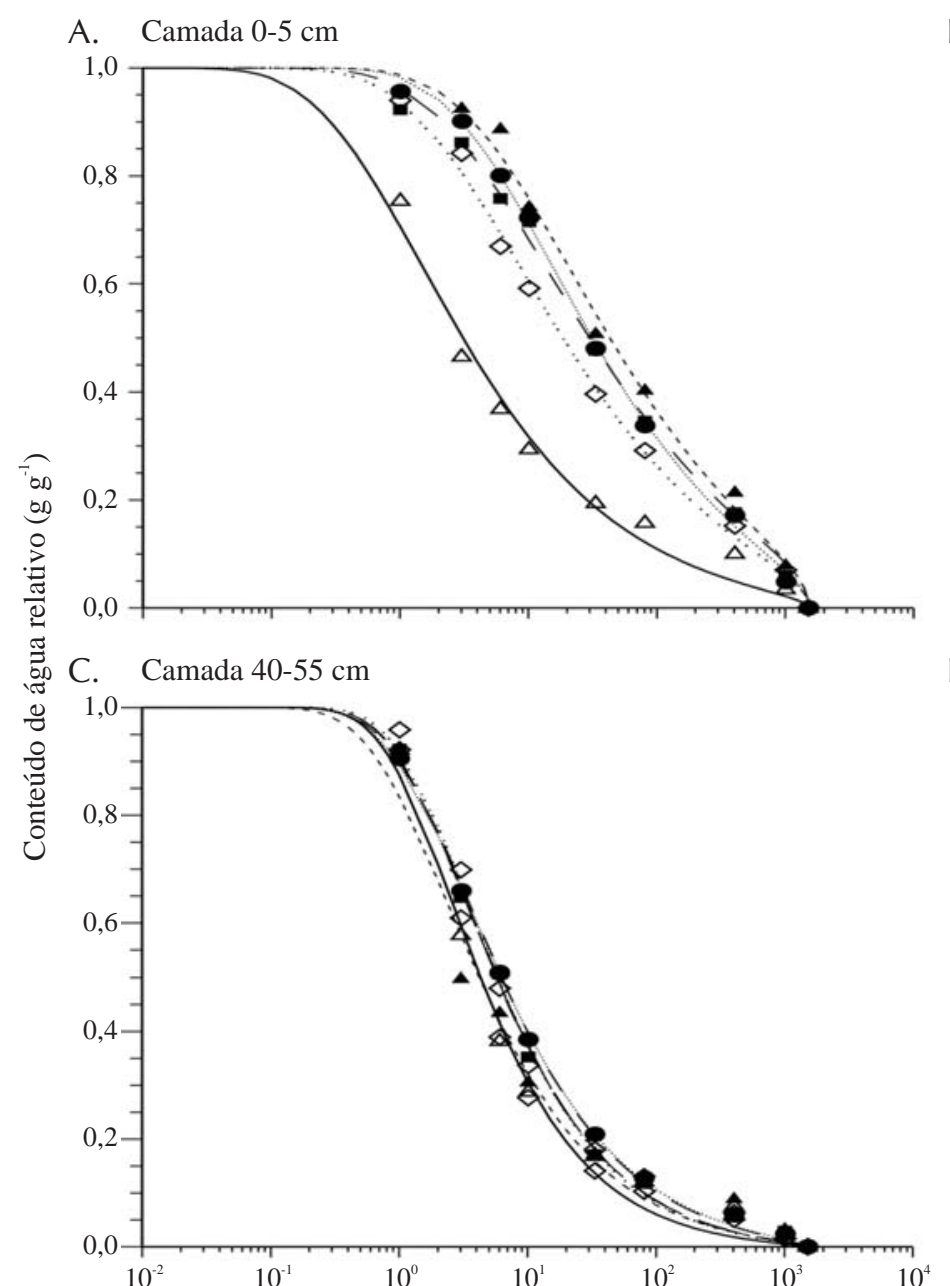

B. Camada $10-15 \mathrm{~cm}$

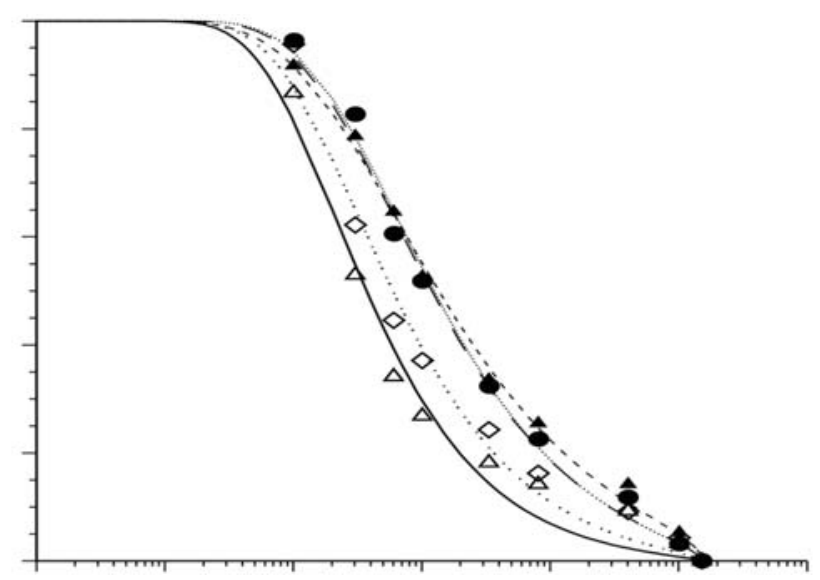

D. Camada $70-80 \mathrm{~cm}$

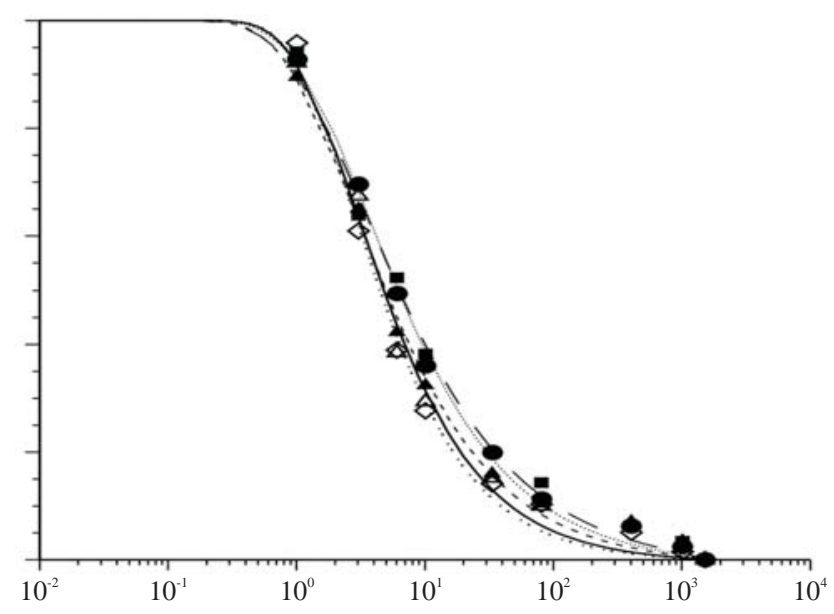

Tensão da água no solo $(\mathrm{kPa})$
$\triangle \quad$ Cn-observado
$\diamond \quad$ Ca-observado
- Pac-observado
A Pmc-observado
- Pbc-observado
Cn-ajuste
Ca-ajuste
- _ Pac-ajuste
...... Pmc-ajuste
Pbc-ajuste

Figura 1. Curvas do conteúdo de água relativo do solo para as camadas: $0-5 \mathrm{~cm}(\mathrm{~A}) ; 10-15 \mathrm{~cm}(\mathrm{~B}) ; 40-55 \mathrm{~cm}(\mathrm{C})$; e $70-80 \mathrm{~cm}(\mathrm{D})$; e para as áreas estudadas: pastagem com alta cobertura (Pac); pastagem com média cobertura (Pmc); pastagem com baixa cobertura (Pbc); cerrado antropizado (Ca) e cerrado natural $(\mathrm{Cn})$

conseqüências aos processos de infiltração e de movimentação de água no solo, com insignificantes efeitos em suas capacidades de armazenamento de água; neste sentido, Fregonezi et al. (2001) verificaram ainda que em solos sob pastagem a estrutura é freqüentemente menos microagregada e menos grumulosa que em solos sob vegetação natural, o que pode resultar em um contato maior entre as partículas, provocando redução da macroporosidade nos espaços inter-agregados e conseqüente aumento da densidade do solo. Alinhado a este resultado, o parâmetro Ma da primeira camada do solo (0-5 cm) foi bastante afetado, com reduções aproximadas de 76, 83, 87 e 92\%, respectivamente, para Ca, Pac, Pbc e Pmc, em relação ao valor de $0,299 \mathrm{~g} \mathrm{~g}^{-1}$ constatado em $\mathrm{Cn}$. Na camada de 10-15 cm, as reduções foram menores mas também expressivas, girando em torno de $40 \%$.

Os dados de Ds variaram entre 0,87 e $1,20 \mathrm{~g} \mathrm{~cm}^{-3}$, valores que podem ser considerados normais para Latossolos (de Maria et al., 1999). Apesar de se ter observado diferenças estatísticas em todas as camadas (Tabela 1), foi nas camadas mais superficiais que se verificaram as maiores varia- ções, enquanto os valores mais altos foram encontrados na camada de $0-5 \mathrm{~cm}$ das áreas de Ca e Pbc (aproximadamente $1,20 \mathrm{~g} \mathrm{~cm}^{-3}$ ), superando em mais de $35 \%$ o patamar referencial do $\mathrm{Cn}\left(0,87 \mathrm{~g} \mathrm{~cm}^{-3}\right)$. Esses valores de densidade podem estar associados ao baixo grau de cobertura vegetal desses solos. Segundo Silva \& Kato (1998), solos descobertos apresentam, ademais, forte redução na taxa de infiltração de água em relação aos protegidos por palha; desta forma, tanto a elevação da Ds quanto a redução da Ma nas camadas superficiais do solo podem estar associadas à baixa cobertura vegetal do solo. A tendência de redução da Ma e do aumento da Ds como resultado do pastejo no solo também foi reportada por Souza et al. (2004), que encontraram baixos valores de Ma (aproximadamente $0,07 \mathrm{~cm}^{3} \mathrm{~cm}^{-3}$ ) na camada de 0-20 cm para um Latossolo Amarelo cultivado com braquiária durante 12 anos, devido à compactação do solo.

Em relação aos resultados sobre a Ks (Tabela 1) verificouse, na camada de 10-15 cm do solo das áreas pastejadas, que houve queda acentuada da condutividade hidráulica relativa ao Cn; no tratamento Ca a redução foi da ordem de 50\% e 
nas pastagens cultivadas de $82 \%$, em média. As áreas sob pastejo se diferenciaram estatisticamente do cerrado natural; já as pastagens cultivadas não apresentaram diferença estatística entre si. Analisando-se o comportamento da Ks ao longo do perfil do solo, notou-se que houve homogeneidade nos seus valores no caso do Cn; nas demais áreas, se constataram diferenças estatísticas entre a camada de $10-15 \mathrm{~cm}$ e aquelas mais profundas evidenciando, assim, a influência do pastejo sobre a infiltração da água em camadas superficiais do solo.

Os resultados de Ks encontrados neste trabalho estão em conformidade com vários estudos já desenvolvidos. Dixon \& Peterson (1971) afirmaram que o tamanho do espaço poroso e sua inter-conectividade determinam a condutividade hidráulica, em que os macroporos são os principais responsáveis pela habilidade da água em se movimentar no perfil do solo, sob condições de quase saturação. Silva \& Kato (1997) notaram que uma pequena redução na Ma é acompanhada de uma grande redução nos valores de con-
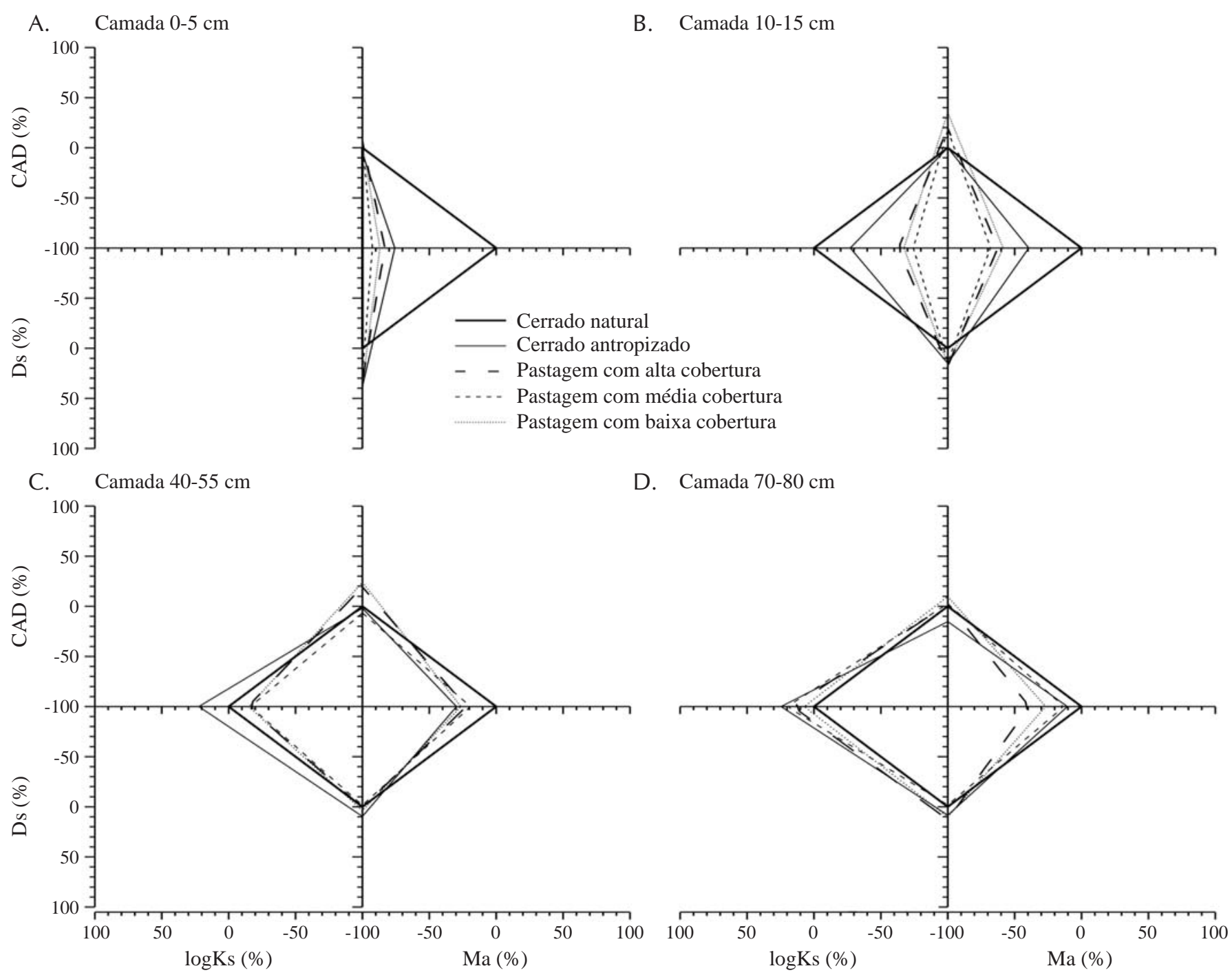

D. Camada $70-80 \mathrm{~cm}$

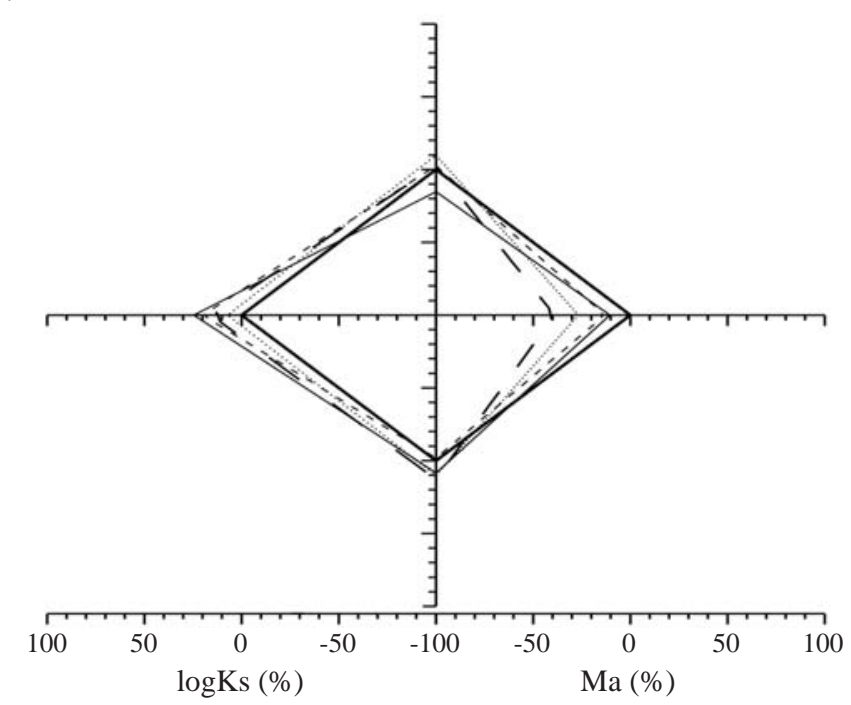

Figura 2. Modelo gráfico comparativo da qualidade físico-hídrica dos solos das áreas estudadas, para as camadas: 0-5 cm (A); $10-15 \mathrm{~cm}(\mathrm{~B}) ; 40-55 \mathrm{~cm}(\mathrm{C})$ e 70-80 cm (D), considerando-se os valores relativos dos parâmetros: capacidade de água disponível (CAD), logaritmo (base 10) da condutividade hidráulica saturada (logKs), densidade do solo (Ds) e macroporosidade (Ma), expressos em percentual em relação ao respectivo valor do parâmetro de referência medido no Cerrado natural

Obs.: A condutividade hidráulica saturada (Ks) não foi avaliada na camada de 0-5 cm devido à impossibilidade de aplicação do tipo de Permeâmetro de Guelph utilizado para essa profundidade dutividade hidráulica. Silva et al. (2003), ao estudarem o comportamento da Ks de um Latossolo de Cerrado sensu strictu nas profundidades de $12 \mathrm{~cm}$ e $80 \mathrm{~cm}$, encontraram um valor médio de $18,52 \mathrm{~cm} \mathrm{~h}^{-1}$ para a menor profundidade e de $21,89 \mathrm{~cm} \mathrm{~h}^{-1}$ para a maior profundidade. Em relação a este último estudo e confrontando os valores de Ks com aqueles obtidos no presente trabalho para o tratamento Ca (Tabela 1), nota-se grande discrepância numérica apenas na menor profundidade, $\mathrm{Ks}=18,52 \mathrm{~cm} \mathrm{~h}^{-1}$ (Silva et al., 2003) e $6,13 \mathrm{~cm} \mathrm{~h}^{-1}$ neste trabalho. Na maior profundidade ocorreu grande semelhança, $21,89 \mathrm{~cm} \mathrm{~h}^{-1}$ (Silva et al., 2003) versus 23,14 $\mathrm{cm} \mathrm{h}^{-1}$ desse ensaio, dando sustentação à idéia de que as atividades do pastejo realmente sonas camadas superficiais do solo desse experimento.

Aplicando-se a técnica da visualização em polígonos (Figura 2) na análise do impacto da atividade de pastejo sobre a qualidade do solo em relação aos atributos da Ds,

\section{B. Camada $10-15 \mathrm{~cm}$}


Ma, CAD e $\operatorname{logKs}$, verificou-se que os polígonos formados pelas pastagens cultivadas foram muito semelhantes entre si. Basicamente, nas duas primeiras camadas do solo existem três formas de polígonos: polígonos muito alongados (pastagens cultivadas), polígonos não tão alongados (cerrado antropizado) e polígonos simétricos (cerrado natural).

Nesta visualização, ficou evidente, nas camadas mais superficiais do solo (Figuras 2A e B), a depreciação da qualidade fisico-hídrica das áreas sob pastejo e Ca, tanto em relação à queda na Ma (Figura $2 \mathrm{~A}$ e B) como na logKs (Figura 2B), indicando que o processo de recuperação da capacidade produtiva das pastagens cultivadas não provocou melhoria na qualidade fisico-hídrica do solo nos três anos de experimento de adubação. O parâmetro físico-hídrico mais sensível ao impacto do pastejo foi a condutividade hidráulica saturada. Em relação aos valores de CAD e de Ds, conforme já discutido, observou-se, visualmente, pouca alteração em relação ao polígono de referência. Nas camadas mais profundas, no entanto, notou-se uma tendência geral dos valores de CAD, Ds, Ma e logKs se aproximarem do polígono de referência.

A apresentação desses resultados nesse tipo de formato gráfico facilita a visualização dos impactos do manejo do solo em sua qualidade, ajudando na tomada de decisão sobre as estratégias a serem adotadas na exploração de determinada área agrícola. No caso desse estudo, um manejo adequado das pastagens deve adotar práticas que proporcionem maior cobertura do solo a fim de minimizar a compactação do pisoteio animal em suas camadas superficiais e seus efeitos negativos na condutividade hidráulica e na macroporosidade do solo.

\section{CONCLUSÕES}

1. O pastejo pouco contribui para o aumento da capacidade de armazenamento da água e a densidade do solo, mas tem impacto significativo na redução da macroporosidade, condutividade hidráulica saturada e do grau de saturação efetiva, nas camadas superficiais do solo (0 a $15 \mathrm{~cm}$ ).

2. Em geral, o Latossolo Vermelho estudado perde em qualidade fisico-hídrica nas camadas de 0-5 e 10-15 cm, em relação ao cerrado natural, em função do uso continuado do pastejo não sofrendo, praticamente, efeito algum nas camadas mais profundas do solo (40 a $80 \mathrm{~cm})$.

\section{AGRADECIMENTOS}

À EMBRAPA, pelo suporte financeiro ao projeto "Desenvolvimento e teste de modelos para estimativa das características físico-hídricas dos solos do Bioma Cerrado" do Macroprograma 2; ao pesquisador Lourival Vilela, da Embrapa Cerrados, pela orientação e apoio no desenvolvimento desta pesquisa.

\section{LITERATURA CITADA}

Assouline, S.; Tessier, D.; Bruand, A. A conceptual model of the soil water retention curve. Water Resources Research, v.34, n.2, p.223-232, 1998.

Bacchi O. O. S.; Reichardt, K. Escalonamento de propriedades hídricas na avaliação de métodos de determinação da condutividade hidráulica de solos. Revista Brasileira de Ciência do Solo, v.12, n.3, p.217-223, 1988.

Balbino, L. C.; Brossard, M.; Stone, L. F.; Bruand, A.; Leprun, J. C. Estrutura e propriedades hidráulicas em latossolos sob cultivo na região do cerrado. Santo Antônio de Goiás: Embrapa Arroz e Feijão, 2003. 43p. Embrapa Arroz e Feijão. Boletim de P\&D, 8.

Barcellos, A. O. Sistemas extensivos e semi-extensivos de produção pecuária bovina de corte nos cerrados. In: Simpósio sobre os Cerrados, International Symposium on Tropical Savannas, 8, 1996, Brasília. Anais... Brasília: Embrapa CPAC, 1996. p.130-136.

Beutler, A. N.; Centurion, J. F.; Silva A. P. da; Roque, C. G.; Ferraz, A. P. da. Compactação do solo e intervalo hídrico ótimo na produtividade de arroz de sequeiro. Pesquisa Agropecuária Brasileira, v.39, n.6, p.575-580, 2004.

Costa, E. A.; Goedert, W. J.; Sousa, D. M. G. Qualidade de solo submetido a sistemas de cultivo com: preparo convencional e plantio direto. Pesquisa Agropecuária Brasileira, v.41, n.7, p.1185-1191, 2006.

de Maria, I. C.; Castro, O. M.; Dias, H. S. Atributos físicos do solo e crescimento radicular de soja em Latossolo Roxo sob diferentes métodos de preparo do solo. Revista Brasileira de Ciências do Solo, v.23, n.3, p.703-709, 1999.

Dias, L. E. Uso de indicadores de qualidade de solo no monitoramento de processos de recuperação de áreas degradadas. Sociedade Brasileira de Ciência do Solo, v.27, n.2, p.6-8, 2002. Boletim Informativo.

Dixon, R. M.; Peterson, A. E. Water infiltration control: A channel system concept. Soil Science American Proceedings, v.35, n.6, p.968-973, 1971.

EMBRAPA - Empresa Brasileira de Pesquisa Agropecuária. Centro Nacional de Pesquisas de Solos. Manual de métodos de análises de solo. 2.ed. Brasília: Embrapa Produção de Informação; Rio de Janeiro: Embrapa Solos, 1997. 212p.

EMBRAPA - Empresa Brasileira de Pesquisa Agropecuária. Centro Nacional de Pesquisa de Solos. Sistema Brasileiro de Classificação de Solos. Brasília. Embrapa Produção da Informação; Rio de Janeiro: Embrapa Solos. 1999. 412p.

Fregonezi, G. A. F.; Brossard, M.; Guimarães, M. F.; Medina, C. C. Modificações morfológicas e físicas de um Latossolo argiloso sob pastagens. Revista Brasileira de Ciência do Solo, v.25, n.4, p.1017-1027, 2001.

Reynolds, W. D.; Elrick, D. E. A laboratory and numerical assessment of the Guelph permeameter method. Soil Science, v.144, n.4, p.282-299, 1987.

Sano, E. E.; Barcellos, A. O.; Bezerra, H. S. Assessing the spatial distribution of cultivated pastures in the spatial distribution of cultivated pastures in the Brazilian savanna. Pasturas Tropicales, v.22, n.3, p.2-15, 2000. 
Silva, C. L.; Kato, E. Efeito do selamento superficial na condutividade hidráulica saturada da superfície de um solo sob cerrado. Pesquisa Agropecuária Brasileira, v.32, n.2, p.213-220, 1997.

Silva, C. L.; Kato, E. Avaliação de modelos para previsão da infiltração de água em solos sob cerrado. Pesquisa Agropecuária Brasileira, v.33, n.7, p.1149-1158, 1998.

Silva, E. M. da; Azevedo, J. A. Influência do período de centrifugação na curva de retenção de água em solos de Cerrado. Pesquisa Agropecuária Brasileira, v.37, n.10, p.1487-1494, 2002.

Silva, E. M. da; Azevedo, J. A.; Rauber, J. C.; Braga, A. R. dos S. Caracterização físico-hídrica e hidráulica de solos do Cerrado submetidos a diferentes sistemas de preparo do solo. Planaltina: Embrapa Cerrados, 2003. 22p. Boletim de P\&D 101.
Silva, E. M. da; Lima, J. E. F. W.; Azevedo, J. A.; Rodrigues, L. N. Valores de tensão na determinação da curva de retenção de água de solos do Cerrado. Pesquisa Agropecuária Brasileira, v.41, n.2, p.323-330, 2006.

Souza, L. D.; Reichardt, K. Estimativas da capacidade de campo. Revista Brasileira de Ciência do Solo, v.20, n.2, p.183189, 1996.

Souza, Z. M.; Leite, J. A.; Beutler, A. N. Comportamento de atributos físicos de um latossolo amarelo sob agroecossistemas do Amazonas. Engenharia Agrícola, v.24, n.3, p.654-662, 2004.

Warrick, A. W. Interrelationships of irrigation uniformity terms. Journal of Irrigation and Drainage Engineering, v.109, n.3, p.317-332, 1983. 